\title{
The Core Membrane Fusion Complex Governs the Probability of Synaptic Vesicle Fusion But Not Transmitter Release Kinetics
}

\author{
Michael F. A. Finley, ${ }^{1,2}$ Sejal M. Patel, ${ }^{1}$ Daniel V. Madison, ${ }^{2}$ and Richard H. Scheller ${ }^{1,2}$

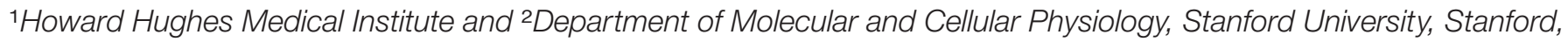 \\ California 94305
}

\begin{abstract}
Synaptic vesicle fusion is driven by the formation of a fourhelical bundle composed of soluble $N$-ethylmaleimide sensitive factor (NSF) attachment protein receptors (SNAREs). Exactly how the structural interactions that lead to the formation of this complex relate to neurotransmitter release is not well understood. To address this question, we used a strategy to "rescue" synaptic transmission after proteolytic cleavage of the synaptosome-associated protein of $25 \mathrm{kDa}$ (SNAP-25) by botulinum neurotoxin $E$ (BoNtE). Transfection of CA3 hippocampal pyramidal cells with BoNtE-resistant SNAP-25 restored synap-
\end{abstract}

tic transmission. Additional mutations that alter the interaction between SNAP-25 C-terminal coil and the other SNARE coils dramatically reduce transmitter release probability but leave the kinetics of synaptic responses unaltered. These data indicate that at synapses, SNARE interactions are necessary for fusion but are not the rate-limiting step of neurotransmission.

Key words: soluble N-ethylmaleimide-sensitive factor (NSF) attachment protein receptors (SNAREs); SNAP-25; botulinum neurotoxin E (BoNtE); hippocampus; synaptic transmission; vesicle fusion
Release of neurotransmitter requires the fusion of synaptic vesicles with the presynaptic plasma membrane. Three proteins, collectively known as soluble $N$-ethylmaleimide sensitive factor (NSF) attachment protein receptors (SNAREs), are required for transmitter release: VAMP (synaptobrevin), located on the vesicle, syntaxin, and SNAP-25, located primarily on the presynaptic membrane (Südhof and Scheller, 2001) (see Fig. 1A). As a critical part of this process, the SNAREs bind together in a parallel, four-helical bundle, with one helix each contributed by VAMP and syntaxin and two contributed by SNAP-25 (Poirier et al., 1998; Sutton et al., 1998), to form an extremely stable, SDSresistant complex that requires NSF-mediated ATP-hydrolysis to be dissociated (Söllner et al., 1993). Each of these helices contains a heptad repeat such that hydrophobic residues from each helix are oriented toward the center of the bundle, contributing to thermal stability (Sutton et al., 1998). In addition, each helix contains a highly conserved inward-facing polar residue at its center (either glutamine or arginine) that contributes to an ionic layer in the middle of the four-helix bundle. It is hypothesized that this ionic layer acts to align the four helices in register with one another (Sutton et al., 1998). The energy released by the formation of this complex may drive the fusion reaction that leads to transmitter release (Hanson et al., 1997; Lin and Scheller, 1997).

Exactly how the structures of the SNARE molecules relate to

\footnotetext{
Received June 27, 2001; revised Oct. 11, 2001; accepted Oct. 22, 2001.

This work was sponsored by the Howard Hughes Medical Institute (R.H.S.) and the Silvio Conte-National Institute of Mental Health Center for Neuroscience Research Grant MH48108 (D.V.M.). We thank T. F. J. Martin for providing the pcDNA3.1-SNAP-25bD179K construct, Yu Chen, Johanna Montgomery, Murali Prakrya, Suzie Scales, Audrey Ettinger, and members of the Scheller and Madison labs for helpful discussions, and Eric Schaible for slice preparation.

Correspondence should be addressed to Daniel V. Madison, Associate Professor, Department of Molecular and Cellular Physiology, Stanford University School of Medicine, Beckman Center, B003, Stanford, CA 94305. E-mail: madison@stanford.edu.

R. H. Scheller's present address: Genentech, Inc., 1 DNA Way, South San Francisco, CA 94080.
}

Copyright (C) 2002 Society for Neuroscience $\quad 0270-6474 / 02 / 221266-07 \$ 15.00 / 0$ their function in synaptic vesicle fusion is under active investigation. Clostridial neurotoxins (Schiavo et al., 2000), highly specific proteases that cleave individual SNARE proteins, have been used extensively as a powerful tool to examine the function of toxincleaved SNARE proteins during neuromuscular or CNS synaptic transmission, as well as for chromaffin cell secretion. In these studies, the investigation of molecular interactions was restricted to the cleavage products produced by these proteases. The role of particular SNARE protein amino acid residues has been investigated by overexpressing mutated forms of the protein in chromaffin cells and monitoring release of norepinephrine from large, dense-core vesicles (Chen et al., 1999; Criado et al., 1999; O'Sullivan et al., 1999; Wei et al., 2000). However, it has been difficult to extend the study of precise molecular interactions among SNAREs to CNS synapses, where neurotransmitter is packaged in small, clear vesicles and transmitter release occurs on a faster time scale. We sought to investigate the structure-function relationship of SNAP-25 in functional mammalian CNS synapses by combining the specificity of clostridial neurotoxin cleavage with the ability to transfect organotypic hippocampal slices with mutated forms of SNAP-25. For the first time, this approach has provided an opportunity to directly examine the effects of SNAP-25 mutants on transmission at a fast, excitatory CNS synapse.

\section{MATERIALS AND METHODS}

Molecular biology. Full-length mouse SNAP-25b containing the toxin resistant mutation $\mathrm{D} 179 \mathrm{~K}$ (GAC to AAG) was expressed using a pcDNA3 (Invitrogen, Carlsbad, CA) mammalian expression vector lacking the neomycin-resistance gene [("pcDNA3m") courtesy of Dr. T. F. J. Martin, University of Wisconsin, Madison, WI]. Additional mutations were made to this construct using PCR-based mutagenesis (QuickChange, Stratagene, La Jolla, CA) and confirmed by sequencing. Epitope-tagged versions of SNAP-25b were made by subcloning SNAP$25 \mathrm{~b}$ from pcDNA3m into a pcDNA3.1(+) (Invitrogen) vector with a multiple cloning site modified to contain the myc sequence N-terminal to SNAP-25. For recombinant protein expression, the full-length SNAP$25 \mathrm{~b}$ constructs were subcloned from pcDNA $3 \mathrm{~m}$ into pGEX and ex- 
pressed as glutathione $S$-transferase fusion proteins. Recombinant botulinum neurotoxin E (BoNtE) light chain (gift of the late Dr. Heiner Niemann, Medizinische Hochschule, Hannover, Germany) was used as described previously (Chen et al., 1999) to test cleavage of wild-type and mutant versions of SNAP-25 in vitro. Wild-type SNAP-25 was partially cleaved $(>50 \%)$ by $10 \mathrm{nM}$ and completely cleaved by $25 \mathrm{~nm}$ BoNtE light chain, whereas no cleavage of BoNtE-resistant SNAP-25 (D179K) was evident even at $400 \mathrm{~nm}$ (data not shown). pEGFP-C1 (Clontech, Palo Alto, CA) was used for expression of green fluorescent protein (GFP).

Biolistic transfection. Organotypic hippocampal slices were prepared as described previously (Pavlidis and Madison, 1999). Slices were transfected using the Helios Gene Gun System (Bio-Rad, Hercules, CA). Parameters were optimized on the basis of previous studies (Wong et al., 1998) and advice from other users and Bio-Rad. Gold beads (1.6 $\mu \mathrm{m}$ diameter) were suspended in $100 \mu \mathrm{l}$ of $50 \mathrm{~mm}$ spermidine. GFP and SNAP-25 expression constructs were added ( $\sim 1 \mu \mathrm{g} \mathrm{DNA} / \mathrm{mg}$ beads) and coprecipitated with $1 \mathrm{M} \mathrm{CaCl}_{2}$ onto gold beads. DNA-bound beads were rinsed three times with $100 \%$ ethanol and resuspended in $100 \%$ ethanol $(1.4 \mathrm{ml} / 10 \mathrm{mg}$ beads) containing $0.02 \mathrm{mg} / \mathrm{ml}$ polyvinylpyrrolidone. The lumen of Tefzel tubing (Bio-Rad) was coated with the DNA-bound gold beads and subsequently cut into 0.5 inch lengths to give "cartridges." The cartridges were stored at $4^{\circ} \mathrm{C}$ with desiccant until time of transfection. Organotypic slices were transfected within $1 \mathrm{hr}$ of preparation. Cartridges were loaded into the Helios Gene Gun, and the DNA-coated gold particles were propelled into the tissue with a short burst of helium pressurized to 100-130 psi. A subset of cells in which a gold particle had penetrated the nucleus expressed the DNA (see Fig. 1). We typically observed one to three GFP-positive pyramidal cells per slice (range, $0-10)$ in addition to a variable number of glial cells and interneurons. Expression of GFP was observed within $24 \mathrm{hr}$ after transfection and lasted for up to 2 weeks.

Immunofluorescence. Slices were fixed in $4 \%$ paraformaldehyde, rinsed three times in PBS, cryoprotected in 30\% sucrose (in PBS), and embedded in Tissue Tek (Sakura, Torrence, CA). Cryostat sections $(15 \mu \mathrm{m})$ of transfected slices were incubated with an antibody against the myc antigen (mouse anti-myc primary 9E10; Santa Cruz Biotechnology, Santa Cruz, CA) at $1: 1000$ for $4-16 \mathrm{hr}$ at $4^{\circ} \mathrm{C}$ and Texas Red anti-mouse secondary (Jackson ImmunoResearch, West Grove, PA) at 1:200 for $1 \mathrm{hr}$ at room temperature. GFP was imaged directly. Slices were mounted in Vectashield, coverslipped, and visualized on a Zeiss Axiophot microscope.

Electrophysiology. Paired, whole-cell patch-clamp recordings in organotypic hippocampal slices were performed as described previously (Pavlidis and Madison, 1999), with minor adjustments. Recordings were made from 6- to 10-d-old cultured slices. Slices were treated with BoNtE holotoxin $(\sim 40 \mu \mathrm{g} / \mathrm{ml}$; Wako) via application of toxin to the culture medium 24-72 hr before the time of recording. Pyramidal cells expressing GFP were targeted as the putative presynaptic cells for whole-cell patch-clamp recording in slices treated with BoNtE. To patch onto pyramidal cells expressing GFP, these cells were first identified in the microscope under fluorescent illumination from a mercury lamp (Zeiss) filtered through an Endow GFP filter set with long-pass emitter (Chroma Technologies, Brattleboro, VT). The gold particle located in the nucleus of the cell [which was visible under both fluorescent and differential interference contrast imaging (DIC)] was subsequently used to relocate the GFP-positive cell after switching to DIC for patch clamping. The presynaptic cell electrode manipulator was attached to the recording stage so that the slice could be moved beneath the objective to locate potential postsynaptic cells. Using this arrangement, multiple postsynaptic cells (typically two to three) were tested for each presynaptic cell. Postsynaptic cells were held at $-85 \mathrm{mV}$ (corrected for junction potential) unless indicated otherwise. Whole-cell patch-clamp recordings were established onto one or more potential postsynaptic partners in the vicinity of the presynaptic cell (100-200 $\mu \mathrm{m}$ away). The presynaptic cell was stimulated to fire action potentials (APs) by passing depolarizing current through the whole-cell electrode at $0.1-0.2 \mathrm{~Hz}$ for $3-5 \mathrm{~min}$ while monitoring the postsynaptic current. If a reliable response that was time locked to the AP was observed, the cell was considered synaptically connected (see Fig. $2 A$, cell 2, B, 1). We confirmed that the response was excitatory by depolarizing the cell to $-55 \mathrm{mV}$, a holding potential at which inhibitory currents become outward using our internal solutions (our unpublished observations). Nontransfected negative control cells were often tested with paired-pulse (see Fig. $2 B, 3$ ) or high-frequency (33-60 Hz; see Fig. 3B, 3, C, 3) bursts of action potentials, or both, to try to draw out potential responses. A minimum of six GFP-negative pairs (two different presynaptic cells, each with three postsynaptic partners) in the same slice as the rescue were tested in this way to rule out the possibility that an apparent rescue might simply be failure of the toxin to block transmission. Approximately $90 \%$ of negative control cells showed no responses to AP stimulation. The remaining $10 \%$ showed failure rates of $>99 \%$ (96 pairs analyzed corresponding to $15 \mathrm{DK}$ rescues). Averages are reported $\pm \mathrm{SE}$. All statistics were performed using Analyze-It software (Leeds, United Kingdom) for Microsoft Excel.

\section{RESULTS}

In this study, we have used four mutants of SNAP-25 designed to alter different functional aspects of this SNARE (Chen et al., 1999). In the first of these mutants (DK), the point mutation D179K was designed to render SNAP-25 resistant to BoNtE cleavage. This mutation is located adjacent to the BoNtE cleavage site (between R180 and I181), and the mutant is at least 40-fold more resistant to BoNtE cleavage than the native SNAP-25 (see Materials and Methods). The other three mutations incorporate the $\mathrm{D} 179 \mathrm{~K}$ mutation to make each of them toxin resistant. The second mutant, D179K/M167A/I171A (MAIA), represents an alteration in the hydrophobicity of the SNAP-25 C-terminal coiled-coil domain by substituting smaller hydrophobic residues (the methyl group of alanine) for the bulky methionine and isoleucine residues found at the -2 and -1 layers of the four-helical bundle. A third mutant, D179K/Q174A (QA), replaces the ionic residue at the central layer of the coiled-coil. Finally, the D179K/K201STOP (STOP) mutant lacks the six C-terminal amino acids, a region of the protein that potentially links SNAP-25 to a calcium-sensing mechanism(s) (Capogna et al., 1997; Trudeau et al., 1998). In addition, this region of the protein contributes to the C-terminal hydrophobic layer closest to the vesicle membrane (Poirier et al., 1998; Sutton et al., 1998).

To address the effects of these SNAP-25 mutants on excitatory, glutamatergic synaptic transmission, we chose the organotypic hippocampal slice preparation because it provides the opportunity to make electrophysiological recordings from individual synaptically connected pairs of neurons that have been transfected with mutant SNAREs. Synaptic transmission between pairs of CA3 pyramidal neurons in organotypic hippocampal slices has been shown previously to be similar, if not identical, to transmission in noncultured tissue (Debanne et al., 1996; Pavlidis and Madison, 1999). These connections between two individual CA3 pyramidal neurons likely consist of an average of $\sim 10$ synapses each (Pavlidis and Madison, 1999). In addition, this preparation is amenable to transfection (Nakayama et al., 2000). BoNtEresistant SNAP-25 (DK) was introduced into organotypic hippocampal slices along with GFP using biolistic-mediated gene transfer. This method typically resulted in one to three GFPpositive pyramidal neurons appearing in each slice (range, $0-10$ ) (Fig. $1 B$ ). Because GFP and SNAP-25 mutants were introduced via the same gold particles but on separate constructs, we confirmed that expression of DK occurred in GFP-positive pyramidal cells by cotransfecting SNAP-25 mutants with an N-terminal myc tag. Immunofluorescent labeling revealed extensive overlap between the expression of GFP and DK, even in fine axon-like processes and varicosities (Fig. 1C). Further mutations in SNAP25, such as those in MAIA and STOP, did not alter the level of expression or the localization of SNAP-25 in pyramidal cells (Fig. $1 C)$. Because the expression levels of GFP and myc-tagged SNAP-25 constructs qualitatively appeared to be correlated, we targeted for study (see below) only those pyramidal cells that strongly expressed GFP.

After transfection with DK and GFP, slices were treated with 

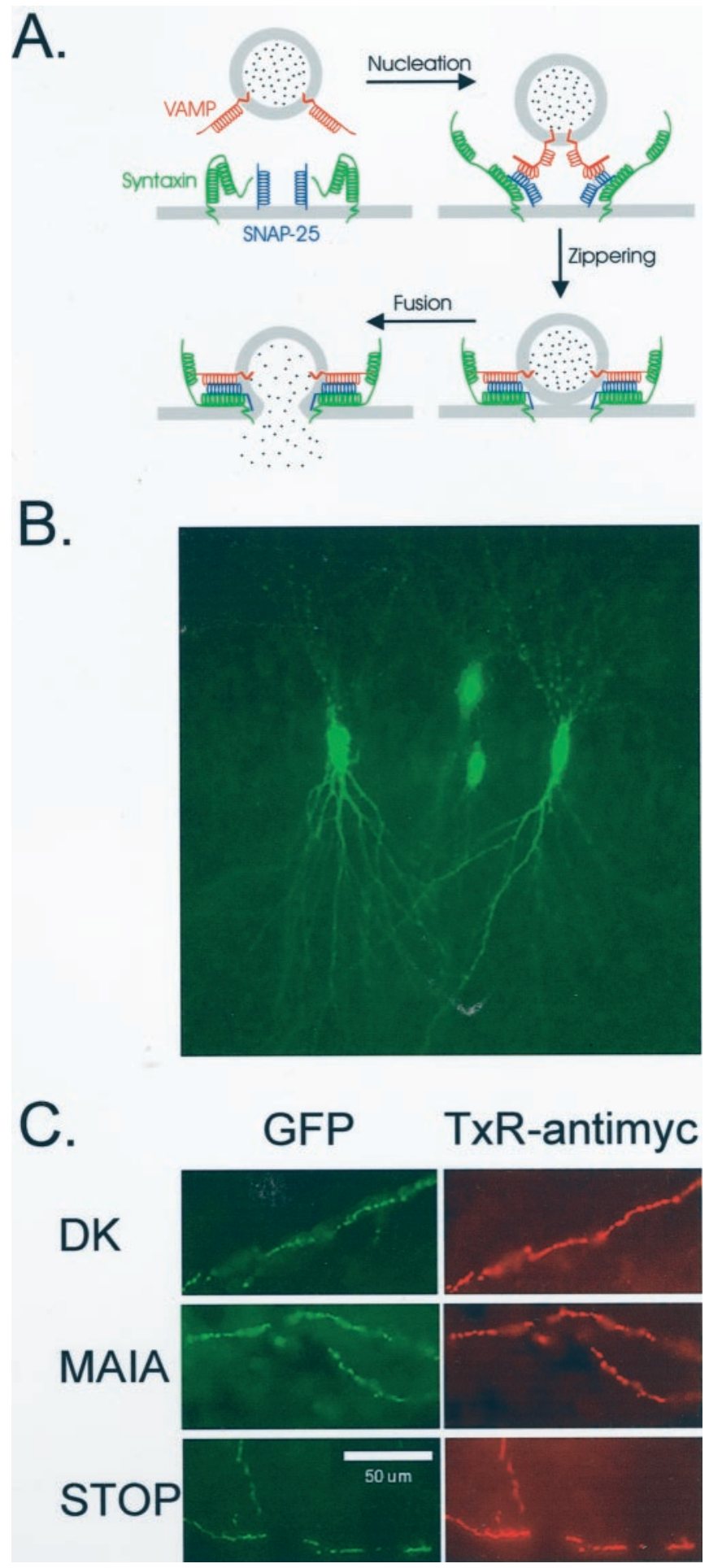

Figure 1. Expression of toxin-resistant SNAP-25 in organotypic hippocampal slices. $A$, Schematic of SNARE interactions leading to synaptic vesicle fusion. VAMP (red), syntaxin (green), and SNAP-25 (blue) are shown. Only one coil of SNAP-25 is shown for purposes of illustration. $B$, CA3 pyramidal cells were transfected with pEGFP by biolistic gene transfer and visualized $5 \mathrm{~d}$ later. $C$, Coexpression of GFP and myc-tagged SNAP-25 mutants in axon-like processes in $15 \mu \mathrm{m}$ cryostat sections of fixed slices 7-9 d in vitro after transfection.

BoNtE (40 $\mu \mathrm{g} / \mathrm{ml}$ BoNtE holotoxin; 24-72 hr) to eliminate the activity of native SNAP-25. Synaptic transmission subsequently was monitored by making simultaneous whole-cell recordings from two CA3 neurons (Debanne et al., 1996; Pavlidis and Madison, 1999). When two cells are connected by active synapses in a nontoxin-treated slice ( $\sim 40 \%$ of all pairs), a presynaptic action potential is followed at short latency by an inward AMPA receptor-mediated EPSC (Debanne et al., 1996; Pavlidis and Madison, 1999). In a BoNtE-treated slice, when the presynaptic cell was not transfected (GFP-negative), paired recordings revealed no synaptic transmission (Fig. $2 A, B, 2,3$ ), indicating that the toxin had effectively cleaved native SNAP-25. However, we did observe synaptic transmission when the presynaptic cell was transfected with DK and GFP (Fig. 2A,B, 1). In 38\% (23 of 61) of presynaptic cells transfected with DK, rescue of transmission occurred with at least one postsynaptic partner. In cells transfected with GFP alone, synaptic transmission was never observed after BoNtE treatment $(n=18$ pairs, $6 \mathrm{GFP}-$ positive/DKnegative presynaptic cells; data not shown). In addition, cells transfected with wild-type SNAP-25 (i.e., lacking the D179K mutation) exhibited no transmission in BoNtE-treated slices ( $n=$ 16 pairs; five GFP-positive/wild-type SNAP-25-transfected presynaptic cells; data not shown).

Synaptic transmission rescued by DK showed similar variability in the EPSC amplitudes as seen in nontransfected, nontoxintreated cell pairs (average $1 / \mathrm{CV}^{2}=7.5$; range from 2.2 to 24.5 ; average peak amplitude of EPSCs ranged from 9 to $47 \mathrm{pA}, 22 \mathrm{pA}$ average) (Fig. 3A,D) (cf. Pavlidis and Madison, 1999). There was also a considerable trial-to-trial failure rate, ranging from 0 to $93 \%$ (average $=41 \pm 6 \% ; n=25$ synaptically connected pairs), comparable to the failure rates observed in native pairs, those in nontransfected slices not treated with BoNtE (average $=34 \pm$ $7 \% ; n=16 ; p>0.05$ ) (Fig. $3 D)$.

Next, we tested the effects of further mutations of SNAP-25 on synaptic transmission. CA3 pyramidal cells transfected with SNAP-25 mutant MAIA or QA showed rescue of transmission with at least one postsynaptic partner in 7 of $24(29 \%)$ or 8 of 21 $(38 \%)$ of cell pairs tested, respectively, within the range of that observed for DK (see above). However, synaptic transmission mediated by MAIA or QA was characterized by a dramatic reduction in synaptic transmission [(Fig. $3 B, D, E)$ and data not shown]. Single action potentials typically evoked few responses in MAIA or QA rescues, demonstrating a significantly higher failure rate relative to DK rescues (Fig. $3 E$ ). To observe an increased number of EPSCs in MAIA and QA rescues, we applied brief high-frequency bursts of APs to the presynaptic cell (Fig. 3B). Brief bursts of APs can increase calcium entry into the presynaptic terminal and increase the probability of neurotransmitter release (Kreitzer and Regehr, 2000). For both MAIA and QA, stimulating the presynaptic cell in this manner resulted in an increase in the number of responses, whereas these bursts did not tend to change the failure rate for DK rescues [(Fig. $3 B, 2)$ and data not shown; failures changed from an average of 85 to $79 \%$ for MAIA, $p=0.07, n=9$; from 88 to $75 \%$ for QA, $p=0.02, n=$ 6; from 57 to $59 \%$ for $\mathrm{DK}, p=0.8, n=7$; paired $t$ tests compared the failure rate at $0.2 \mathrm{~Hz}$ with the failure rate during brief pulses of three to five APs at 20-60 Hz, respectively). STOP-mediated rescue displayed an even more severe reduction in neurotransmission. To observe any EPSCs with this mutant, paired-pulse stimulation or application of high external calcium concentrations, or both, was almost always necessary (Fig. 3C) (cf. Capogna et al., 1997; Trudeau et al., 1998). Although DK rescues typically showed paired-pulse depression, STOP rescues typically displayed facilitation with paired-pulse stimulation [average log $($ EPSC2/EPSC1 $)=1.3 \pm 0.4$ for STOP and $=-0.15 \pm 0.2$ for 
A.

$$
\text { postsynaptic }
$$

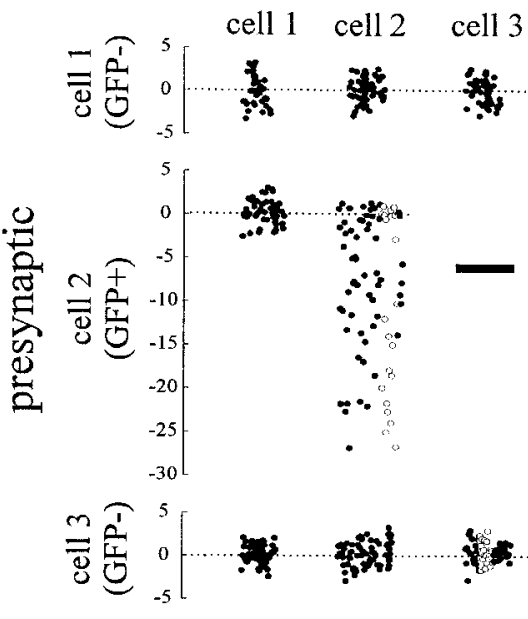

B.
GFP+

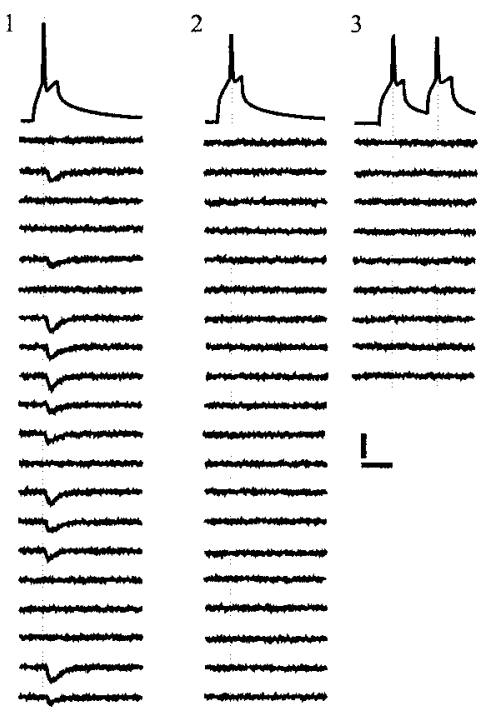

Figure 2. Toxin-resistant SNAP-25 can restore transmitter release in the presence of BoNtE. $A$, In a typical experiment, a putative presynaptic pyramidal cell can be tested with a series of potential postsynaptic partners. APs were stimulated at $0.2 \mathrm{~Hz}$ for $3-5 \mathrm{~min}$ in the putative presynaptic cell at the same time that the peak current of the potential postsynaptic cell (in picoamperes; 0 ) was measured within a short (1-10 $\mathrm{msec}$ ) window after the peak of the AP. In this experiment, the GFP+ presynaptic cell (cell 2) evoked monosynaptic EPSCs in the second postsynaptic partner tested. GFP- presynaptic cells (cells 1 and 3) from the same slice as the rescue were tested with three putative postsynaptic partners each and serve as negative controls. No EPSCs were observed in these pairs. Calibration: $5 \mathrm{~min}$. $B$, Consecutive postsynaptic currents (20 traces; $\bigcirc$ indicates corresponding amplitudes in $A$ ) from the rescue pair are shown (1). EPSCs are evident as downward deflections of the current trace occurring shortly after the AP peak. Negative control consecutive traces show no EPSCs in response to either a single presynaptic AP (2) or paired-pulse stimulation (3) from the third putative postsynaptic partner of presynaptic cell 3 (see corresponding amplitudes in $A, \bigcirc$ ). Calibration: $50 \mathrm{pA}, 25 \mathrm{msec}$.

DK; $p<0.01$; independent samples $t$ test; $n=5$ and 10, respectively]. The average peak amplitudes of EPSCs for MAIA, QA, and STOP rescues were each reduced relative to DK rescues and native recordings (Fig. 3D) (Native indicates data from paired recordings from nontransfected, nontoxin-treated slices). The trial-to-trial failure rate was significantly increased in MAIA, $\mathrm{QA}$, and STOP rescues in comparison to DK rescue and native transmission (Fig. 3E), suggesting that fewer neurotransmitter release events were stimulated by each presynaptic action potential.

The effects of expression of STOP and MAIA in the absence of toxin treatment were also tested. Pairs of CA3 cells in which the presynaptic cell expressed STOP showed an impairment of synaptic transmission relative to native (untransfected) pairs in the absence of BoNtE treatment. (Fig. $3 F$ ) (failure rates of $34 \pm 7 \%$ and $82 \pm 9 \%$ for native and STOP, respectively; $p<0.01 ; n=16$ and 5 , respectively). In contrast, when we tested MAIA in the absence of BoNtE treatment, we were unable to detect a significant reduction in transmission (failure rate of $31 \pm 18 \% ; n=5$ pairs).

Although the probability of release is reduced with MAIA, $\mathrm{QA}$, and STOP rescues relative to DK, we sought to determine whether the nature of transmission, when it did occur, was altered by these mutations. We compared kinetic parameters of EPSCs from DK, QA, MAIA, and STOP transfected cells and those of native pairs, including average $10-90 \%$ rise time and the $\tau_{\text {decay. }}$. We found no differences in these kinetic parameters among any of these conditions (Fig. $4 A, B$ ). In addition, there was no significant difference in the latency to onset of the EPSC (Fig. 4C).

\section{DISCUSSION}

Our study directly investigates, for the first time, the effects of specific amino acid mutations in SNAP-25 on neurotransmitter release in a functional mammalian CNS synapse. Critical to this study was the use of a BoNtE-resistant form of SNAP-25, namely the D179K mutation. Previous studies have demonstrated that SNAP-25 rendered resistant to cleavage by BoNtA (O'Sullivan et al., 1999) and BoNtE (Washbourne et al., 1999) can support neurosecretion in chromaffin and PC12 cells, respectively. Similarly, neither GFP alone nor wild-type SNAP-25 overexpression was able to restore transmission in our study, indicating that expression of BoNtE-resistant SNAP-25 is solely responsible for restoring synaptic transmission in the presence of nonfunctional native SNAP-25. In addition, our observations demonstrated that synaptic transmission supported by DK shares the same basic properties of transmission as observed under native conditions in this preparation.

Previous studies in chromaffin cells or PC12 cells have tested MAIA (Chen et al., 1999), mutations of Q174 (Chen et al., 1999; Wei et al., 2000), and K201STOP (Criado et al., 1999). Our results are consistent with those observations in that we saw an effective reduction of transmission with these mutants (Fig. $3 B-D$ ). Most of these studies draw conclusions based on the fusion of many (tens to hundreds) vesicles over the course of seconds to tens of minutes, where it is difficult to directly observe whether release of individual vesicles is impaired by a reduction in the probability of vesicle fusion or by a slowing of release kinetics, or both [but see Criado et al. (1999)]. One of the greatest advantages of our approach is the ability to measure the kinetics of transmission at a limited population of CNS synapses with millisecond resolution in response to a precise stimulus. Our data suggest that mutations that disrupt, even subtly (e.g., MAIA), interactions between the SNARE coiled coils, have profound effects on the probability of vesicle fusion (Fig. 3) but do not alter the kinetics of transmitter release or the time to onset of the fusion event (Fig. 4).

Either overexpression of SNAP-25 lacking the last nine amino 
A.
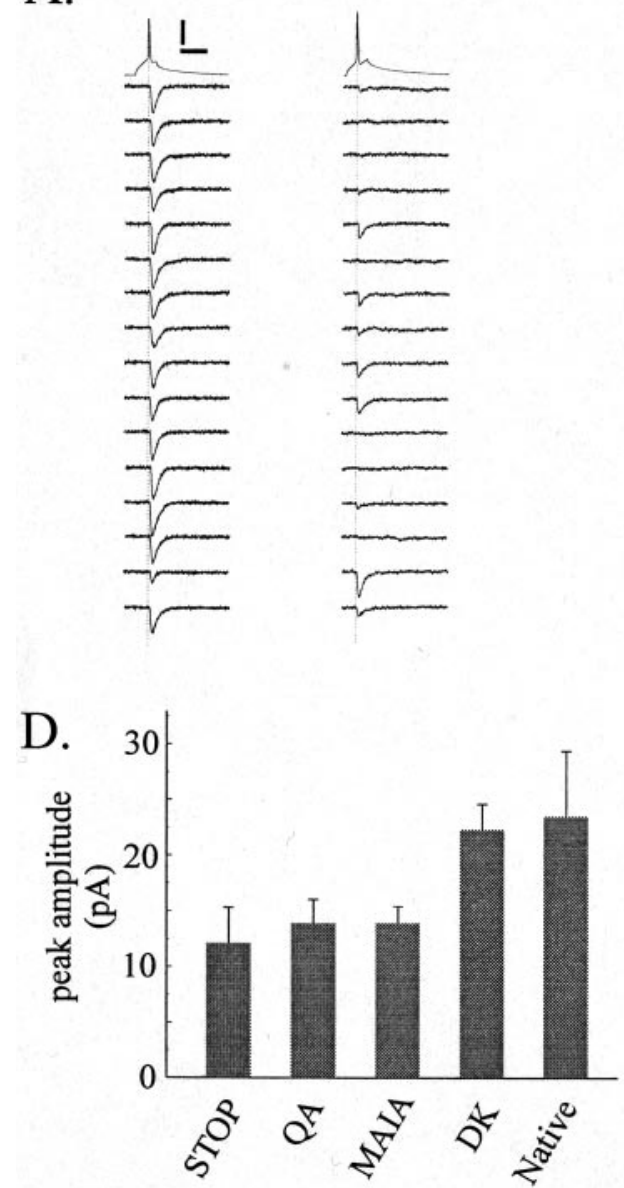

B.

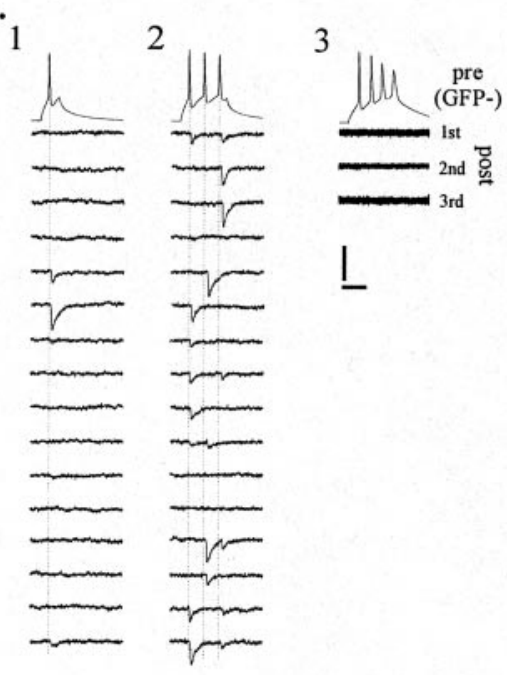

C.
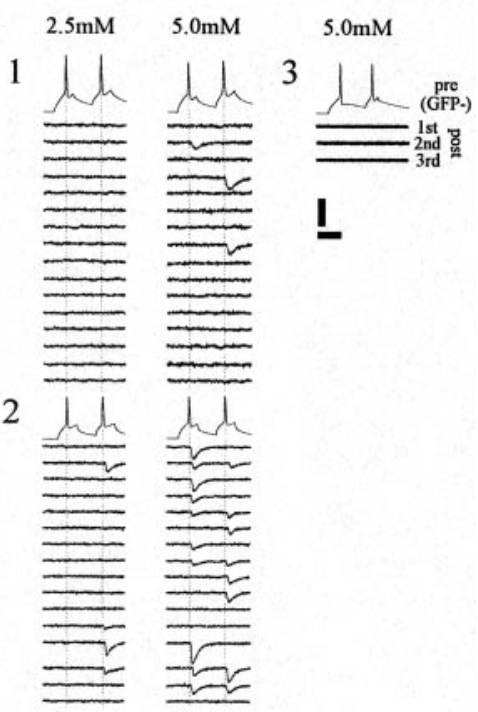

F. $1 \underset{\text { no toxin }}{\text { GFP- }}$
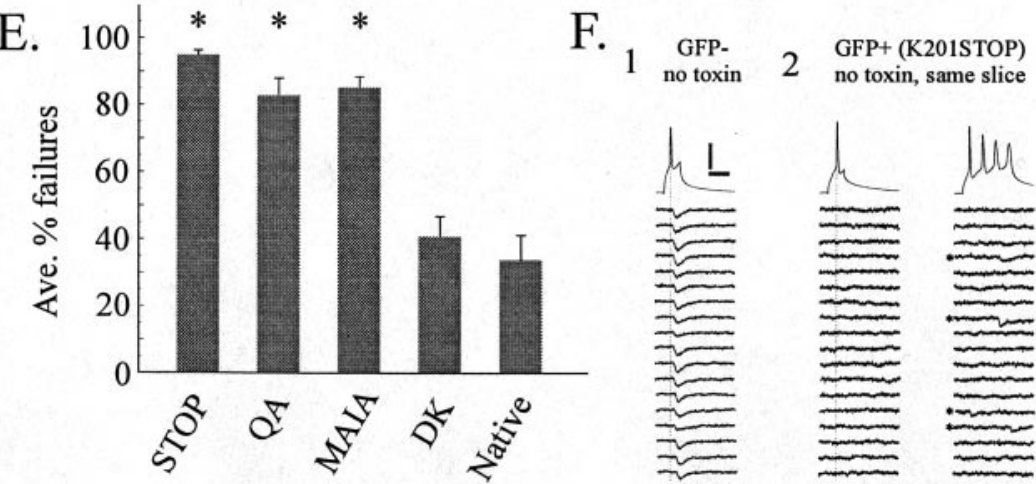

Figure 3. Mutations that affect the interaction of SNAP-25 C terminus with other SNAREs reduce transmitter release. $A$, Two representative examples (not including the example in Fig. 2) of DK rescue of synaptic transmission. Consecutive postsynaptic responses from each rescue are shown. $B$, Consecutive traces from one MAIA rescue. Responses to single AP (1) or a brief burst of APs (at $60 \mathrm{~Hz} ; 2)$ are shown. Superimposed consecutive traces $(\sim 20)$ from three different putative postsynaptic partners of a single GFP - presynaptic pyramidal cell stimulated to fire $60 \mathrm{~Hz}$ bursts of APs $(3)$. Cells are from the same slice as the rescue. $C$, Consecutive traces from two different $(1,2)$ STOP rescues recorded in Ringer's solution containing either 2.5 or $5 \mathrm{~mm}$ extracellular $\mathrm{Ca}^{2+}$. Paired-pulse stimulation was used to observe responses. Negative control (GFP- presynaptic cell, 3 ) from the same slice as 1 showing no responses to paired-pulse stimulation in the presence of $5 \mathrm{mM} \mathrm{Ca}^{2+}$ Ringer's solution in any of its three postsynaptic partners (30 consecutive traces superimposed for each). $D$, An average of mean peak amplitudes ( \pm SE; the mean for each rescue was determined from 5-40 EPSCs); $E$, percentage failures $(100 \times$ number of failures/total number of stimulations at $0.2 \mathrm{~Hz} ; \sim 50$ trials per rescue $)$ for all rescues of the given mutation $(n=$ $5,8,9,23$, and 16 pairs for STOP, QA, MAIA, DK, and native pairs, respectively). MAIA, QA, and STOP mutant rescues each have significantly higher failure rates compared with DK rescues and native pairs (one-way between-subjects ANOVA; $p<0.0001$; with all pair-wise contrasts using Tukey error protection at $99 \%$ confidence interval; * indicates significance when compared with DK or native). Average peak amplitudes tended to be higher for DK and native pairs relative to the other mutants, although not significantly ( $p=0.2$; one-way between-subjects ANOVA). Note that values for STOP mutants were taken from responses during paired-pulse stimulation and not single APs as for native, DK, MAIA, and QA (see Results). F, Expression of STOP in the absence of toxin acts to reduce transmission. Consecutive traces from a control cell (1) and a STOP-expressing cell (2) from the same slice. Note that this STOP expressing cell shows responses (*) only with a train of action potentials. Calibration $(A-C, F): 50 \mathrm{pA}, 25 \mathrm{msec}$.

acids in chromaffin cells (Wei et al., 2000) or treatment of dissociated hippocampal neurons with botulinum neurotoxin A (but see Capogna et al., 1997; Trudeau et al., 1998), which removes the last nine amino acids of SNAP-25, resulted in a delay to onset of vesicle release in addition to significantly reduced levels of vesicle fusion. Our results with rescue mediated by the STOP mutant (lacking the last six amino acids), where we detected a severe reduction in vesicle fusion events (Fig. $3 B, E$ ) but no change in onset to fusion (Fig. $4 C$ ), may suggest that the three additional amino acids (198-200) are enough to keep the fusion event time-locked to the presynaptic trigger.

Interestingly, overexpression of STOP in the absence of BoNtE resulted in reduced transmission (Fig. $3 F$ ) relative to transmission between native (untransfected) pairs, whereas MAIA was unable to inhibit release in this manner. The MAIA mutation occurs in the N-terminal half of the $\mathrm{C}$-terminal coil, whereas the STOP mutation deletes the most C-terminal amino acids of this coil, closest to the vesicle (Poirier et al., 1998; Sutton et al., 1998). In a zippering model of fusion (Lin and Scheller, 1997), the SNARE coils come together at their N-terminal ends first, then "zipper up" to the C termini. MAIA may be less able to form initial "loose" SNARE complexes, which may require N-terminal interactions, and is therefore unable to compete with functional, native SNAP-25 to get into SNARE complexes. In contrast, STOP can enter SNARE complexes in the presence of functional, native SNAP-25, but it does not zipper completely because of the C-terminal deletion.

Our results indicate that the stability of the SNARE coiled-coil 

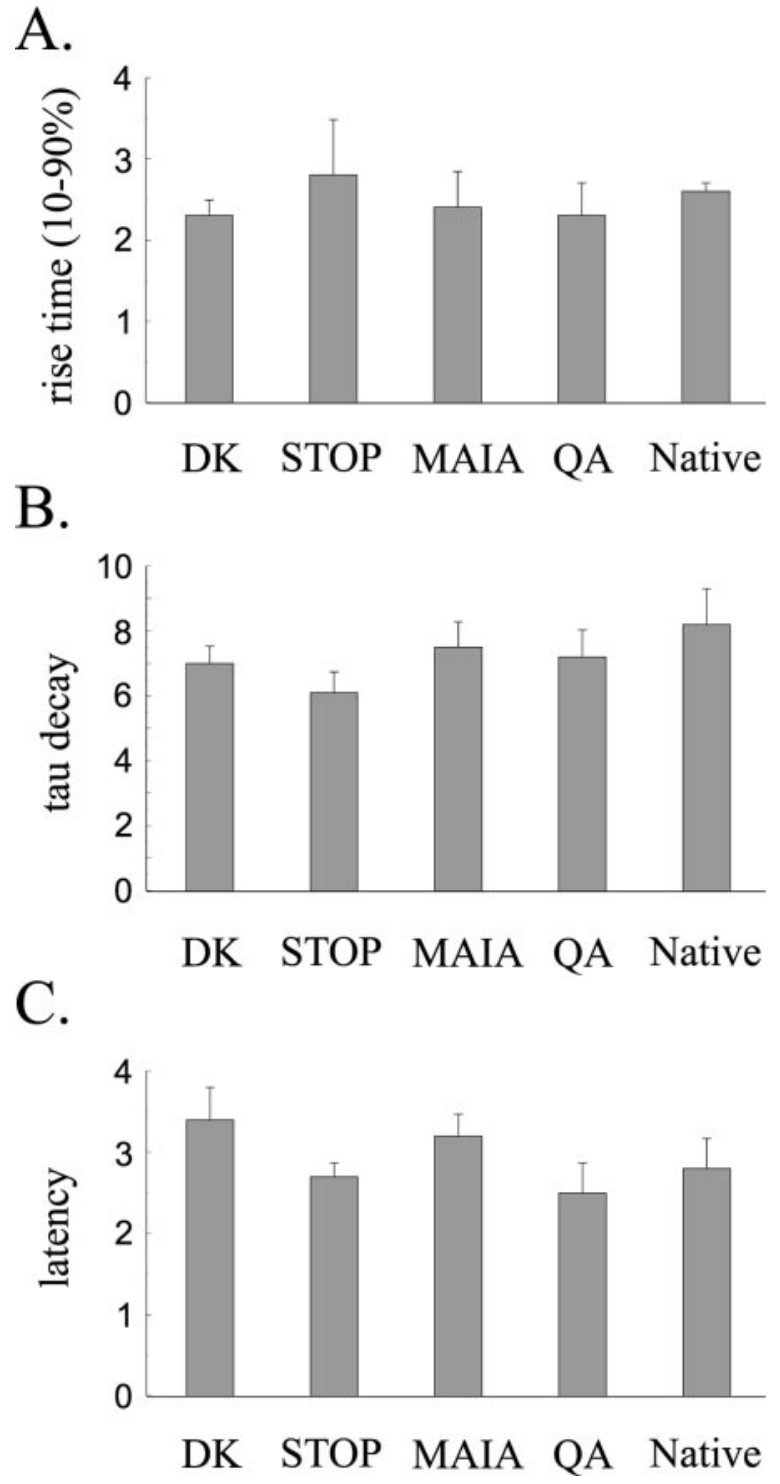

Figure 4. Kinetics of EPSCs are not significantly different among SNAP-25 mutation rescues. Comparisons of average 10-90\% rise time $(A), \tau_{\text {decay }}$ (for single exponential fit) $(B)$, and latency $(C)$ given in milliseconds for DK, STOP, MAIA, and QA rescues and native recordings $(n=23,5,9,8$, and 10 pairs, respectively). EPSC kinetics were analyzed using Mini Analysis Program (Synaptosoft Inc., Leona, NJ). The $10-90 \%$ rise time and the $\tau_{\text {decay }}$ were determined for each rescue by analyzing an average waveform composed of 7-47 EPSCs. Latency was determined by measuring the delay between the peak of the AP and the onset of the EPSC using custom software developed in Labview (National Instruments) (Pavlidis and Madison, 1999). No statistical difference was found among any of the SNAP-25 mutant rescues or native pairs in latency $(p=0.6), 10-90 \%$ rise time $(p=0.75)$, or $\tau_{\text {decay }}(p=0.64)$ using a one-way between subjects ANOVA.

interactions is important in determining the probability that an action potential will promote neurotransmitter release, but that this complex does not regulate the onset or the kinetics of the EPSC once it is initiated. In the context of our data, the following models are plausible. First, the mutants (MAIA, QA, and STOP) may reduce the ability of the SNAP-25 C terminus to nucleate with the other SNAREs, leading to reduced zippering/fusion events or a reduction of, at any given time, the number of complexes available to zipper in response to an action potential.
Second, the mutants may not interfere with nucleation, but instead may impair the ability of the SNARE coiled coil to zipper stably. When complete zippering does occur, fusion occurs at the same rate. Third, a final rate-limiting step before fusion may be downstream of zippering. In this case, the mutants may provide fewer zippered complexes and/or may increase the energy barrier for the zippered complexes to transition to this final rate-limiting step.

Our study may also have interesting implications for the potential role of SNAP-25 (and the SNARE complex in general) in postsynaptic delivery of AMPA receptors. Recent studies have indicated that either disruption of NSF interactions (Lüthi et al., 1999) or cleavage of VAMP postsynaptically with botulinum neurotoxin B (Lüscher et al., 1999) can reduce the basal AMPA receptor-mediated EPSC on the time scale of minutes, reaching a lower steady-state level. A continual supply of AMPA receptors seems to be necessary for a sizable portion of the basal AMPA synaptic current (Lüscher et al., 1999; Lüthi et al., 1999; Kim and Lisman, 2001). In our study, the postsynaptic cell has been exposed to botulinum neurotoxin over the course of 1-2 $\mathrm{d}$. The fact that we see any rescue of transmission at all is consistent with the idea that there exists a pool of AMPA receptors that is either stable in the membrane or does not require SNAREs for movement into or out of the postsynaptic membrane. In our study, peak conductances (used to correct for differences in holding potential) of DK rescues showed no significant difference from those of native pairs $(0.26 \pm 0.03 \mathrm{nS}, n=26$ pairs, and $0.33 \pm 0.09$ $\mathrm{nS}, n=15$ pairs, respectively; $p \gg 0.05$; independent sample $t$ test). This observation may suggest that compensatory mechanisms exist to keep the "stable" receptor pool at a certain size over the long term.

\section{REFERENCES}

Bruns D, Jahn R (1995) Real-time measurement of transmitter release from single synaptic vesicles. Nature 377:62-65.

Capogna M, McKinney RA, O'Connor V, Gahwiler BH, Thompson SM (1997) $\mathrm{Ca}^{2+}$ or $\mathrm{Sr}^{2+}$ partially rescues synaptic transmission in hippocampal cultures treated with botulinum toxin $\mathrm{A}$ and $\mathrm{C}$, but not tetanus toxin. J Neurosci 17:7190-7202.

Chen YA, Scales SJ, Patel SM, Doung YC, Scheller RH (1999) SNARE complex formation is triggered by $\mathrm{Ca} 2+$ and drives membrane fusion. Cell 97:165-174.

Criado M, Gil A, Viniegra S, Gutiérrez LM (1999) A single amino acid near the $\mathrm{C}$ terminus of the synaptosome associated protein of $25 \mathrm{kDa}$ (SNAP-25) is essential for exocytosis in chromaffin cells. Proc Natl Acad Sci USA 96:7256-7261.

Debanne D, Guerineau NC, Gahwiler BH, Thompson SM (1996) Paired-pulse facilitation and depression at unitary synapses in rat hippocampus: quantal fluctuation affects subsequent release. J Physiol (Lond) 491:163-176.

Hanson PI, Roth R, Morisaki H, Jahn R, Heuser JE (1997) Structure and conformational changes in NSF and its membrane receptor complexes visualized by quick-freeze/deep-etch electron microscopy. Cell 90:523-535.

Kim C-H, Lisman JE (2001) A labile component of AMPA receptormediated synaptic transmission is dependent on microtubule motors, actin, and $N$-ethylmaleimide-sensitive factor. J Neurosci 21:4188-4194.

Kreitzer AC, Regehr WG (2000) Modulation of transmission during trains at a cerebellar synapse. J Neurosci 20:1348-1357.

Lin RC, Scheller RH (1997) Structural organization of the synaptic exocytosis core complex. Neuron 19:1087-1094.

Lüscher C, Xia H, Beattie EC, Carroll RC, von Zastrow M, Malenka RC, Nicoll RA (1999) Role of AMPA receptor cycling in synaptic transmission and plasticity. Neuron 24:649-658.

Lüthi A, Chittajallu R, Duprat F, Palmer MJ, Benke TA, Kidd FL, Henley JM, Isaac JTR, Collingridge GL (1999) Hippocampal LTD expression involves a pool of AMPARs regulated by the NSF-GluR2 interaction. Neuron 24:389-399.

Nakayama AY, Harms MB, Luo L (2000) Small GTPases Rac and Rho in the maintenance of dendritic spines and branches in hippocampal pyramidal neurons. J Neurosci 20:5329-5338. 
O'Sullivan GA, Mohammed N, Foran PG, Lawrence GW, Dolly JO (1999) Rescue of exocytosis in botulinum toxin A-poisoned chromaffin cells by expression of cleavage-resistant SNAP-25. Identification of the minimal essential C-terminal residues. J Biol Chem 274:36897-36904.

Pavlidis P, Madison DV (1999) Synaptic transmission in pair recordings from CA3 pyramidal cells in organotypic culture. J Neurophysiol 81:2787-2797.

Poirier MA, Xiao W, Macosko JC, Chan C, Shin YK, Bennett MK (1998) The synaptic SNARE complex is a parallel four-stranded helical bundle. Nat Struct Biol 5:765-769.

Schiavo G, Matteoli M, Montecucco C (2000) Neurotoxins affecting neuroexocytosis. Physiol Rev 80:717-766.

Söllner T, Bennett MK, Whiteheart SW, Scheller RH, Rothman JE (1993) A protein assembly-disassembly pathway in vitro that may correspond to sequential steps of synaptic vesicle docking, activation, and fusion. Cell 75:409-418.

Südhof TC, Scheller RH (2001) Mechanism and regulation of neuro- transmitter release. In: Synapses (Cowan WM, Südhof TC, Stevens CF, eds), pp 177-216. Baltimore: Johns Hopkins University.

Sutton RB, Fasshauer D, Jahn R, Brünger AT (1998) Crystal structure of a SNARE complex involved in synaptic exocytosis at $2.4 \AA$ resolution. Nature 395:347-353.

Trudeau LE, Fang Y, Haydon PG (1998) Modulation of an early step in the secretory machinery in hippocampal nerve terminals. Proc Natl Acad Sci USA 95:7163-7168.

Washbourne P, Boroletto N, Graham ME, Wilson MC, Burgoyne RD, Montecucco C (1999) Botulinum neurotoxin E-insensitive mutants of SNAP-25 fail to bind VAMP but support exocytosis. J Neurochem 73:2424-2433.

Wei S, Xu T, Ashery U, Kollewe A, Matti U, Antonin W, Rettig J, Neher E (2000) Exocytotic mechanism studied by truncated and zero layer mutants of the C-terminus of SNAP-25. EMBO J 19:1279-1289.

Wong WT, Sanes JR, Wong RO (1998) Developmentally regulated spontaneous activity in the embryonic chick retina. J Neurosci 18:8839-8852. 